\title{
CORRADO SEGRE, GUIDO CASTELNUOVO AND THE RIEMANN-ROCH THEOREM
}

\author{
EDOARDO SERNESI
}

\section{INTRODUCTION}

Almost all the mathematical work of C. Segre has been collected in four volumes published by Unione Matematica Italiana between the 1950's and the 1960's. The ordering of the material is not chronological, but obeys some criterion of homogeneity decided by the Editors. In particular the first volume collects those papers considered by them as the most characteristic and important. This is confirmed by F. Severi in the Preface to volume I [8]. This is a short but intense contribution in which he explains carefully the originality of Segre's work. In his energetic and colourful style Severi is generous in giving praises to his master, as well as disapproval to unmentioned addressees. One example will suffice here. On p. VII Severi writes:

Quando i concetti hanno vissuto quasi un secolo è difficile per chi li possiede d'imaginare lo sforzo che i pionieri dovettero compiere per impadronirsene. Occorre all'uopo sobbarcarsi ad una fatica di ricostruzione critica, alla quale oggi si dà scarsissimo peso, essendo diffuso il vezzo di ridurre quasi a zero la bibliografia e la prospettiva storica, le quali evidentemente costano molto lavoro di consultazione, di comparazione e di riflessione, che pur sarebbe sempre onesto ed utile di compiere!

It is interesting to compare Severi's view with today's perception of Segre's legacy. It is true that what was called the hyperspatial method ("il metodo iperspaziale") in algebraic geometry is so natural and obvious today that it does not even deserve to be called a "method". It is less obvious to recall that Segre pionereed the study of vector bundles on algebraic curves and of rational ruled varieties. It is worth observing how important and fashionable these varieties are today, since they play a central and yet not completely

disclosed role in all those investigations dealing with equations and syzygies of projective varieties. Perhaps they represent today the most effective sign of Segre's impact.

It is also interesting to read from Severi that one of the main goals, if not achievements, of the early geometers, including Brill and Noether, was to geometrize algebra rather than considering geometry as a part of algebra. This clear cut statement is somehow disputable today, when we have such a ramified and complex interaction of algebraic geometry with algebra in a 
wide sense. Perhaps Segre himself would agree to a compromise, after seeing the astonishing interplay between equations, syzygies and geometry that are being discovered today.

In this note I want to focus on the hyperspatial method and to explain how the theory of algebraic curves was conceived from this point of view. In 1873 the epoch making memoir [2] of Brill and Noether had appeared; here they introduced the notion of linear series and used plane curves to give a rigorous foundation to the theory. After the early work of Veronese and Bertini, the possibility of interpreting the theory of linear series on a curve as the geometry of curves in a projective space of arbitrary dimension gradually emerged with C. Segre. One of the outcomes of this hyperspatial point of view was finally a projective proof of the Riemann-Roch theorem, which used as main tools the geometry of ruled varieties and some enumerative formulas, originally due to Chasles and Zeuthen, to replace what in Brill and Noether was represented by the so-called "Fundamentalsatz" $A f+B \varphi$. This project of Segre is explained in detail in [6] and was brought to a final form by Castelnuovo in [3], where he used a formula of Schubert's as the only enumerative tool. The proof includes the definition of the canonical series, of the genus, and the deduction of the Riemann-Roch formula, including the duality statement in its numerical form, i.e. the identification of the index of speciality as the vector space dimension of the residual series. Even the proof of the enumerative formula was given from scratch. Here I will reproduce the elegant one given by Enriques in [4], but it can be also found for example in [7].

For the convenience of the reader I will recount the entire story from the beginning, using the modern language of sheaves and cohomology. Despite the apparently technical language, nothing besides elementary projective geometry will be used.

\section{The CANONICAL LINEAR SERIES AND THE GENUS}

We will denote by $C$ a projective irreducible and nonsingular curve defined over $\mathbb{C}$. Let $D=\sum n_{i} p_{i} \in \operatorname{Div}(C)$ be a divisor on $C$.

A vector subspace $V \subset H^{0}(C, \mathcal{O}(D))$ of dimension $r+1 \geq 1$ defines a set of effective divisors linearly equivalent to $D$

$$
|V|=\{\operatorname{div}(\sigma): \sigma \in V, \sigma \neq 0\}
$$

which is canonically identified with the projective space $\mathbb{P}(V)$; we call $|V|$ the linear series defined by $V$, of degree $n=\operatorname{deg}(D)$ and dimension $r$. If $V=H^{0}(C, \mathcal{O}(D))$ then $|V|$ will be denoted by $|D|$ and called the complete linear series associated to $D$ : it is the set of all effective divisors which are linearly equivalent to $D$. For an invertible sheaf $L$ we will write $|L|$ to denote the complete linear series $\left|H^{0}(C, L)\right|$. If $\Delta$ is an effective divisor and $V \subset H^{0}(L)$ we let

$$
V(-\Delta)=V \cap H^{0}(L(-\Delta))
$$


Similarly, $V(\Delta) \subset H^{0}(L(\Delta))$ denotes the image of $V$ under $H^{0}(L) \longrightarrow$ $H^{0}(L(\Delta))$. The symbol $g_{n}^{r}$ is a synonimous for "linear series of dimension $r$ and degree $n "$.

Let $|V|$ be a base-point-free (shortly bpf) $g_{n}^{1}$ and let $J_{V}$ be its jacobian divisor, namely the ramification divisor of the corresponding morphism $\varphi_{V}$ : $C \longrightarrow \mathbb{P}^{1}$.

Proposition 2.1. (i) If $|D|$ is a bpf $g_{n}^{r}, r \geq 2$, then the jacobian divisors of all the bpf $g_{n}^{1}$ 's contained in it are linearly equivalent.

(ii) If $|D|$ is a bpf $g_{n}^{r}$ and $|E|$ is a bpf $g_{m}^{s}$ then

$$
\left|J_{D+E}\right|=\left|J_{D}+2 E\right|=\left|J_{E}+2 D\right|
$$

where $J_{D}, J_{E}, J_{D+E}$ are the jacobian divisors of bpf pencils arbitrarily chosen in $|D|,|E|,|D+E|$ respectively.

Proof. (i) It suffices to prove it for any two bpf pencils contained in a $g_{n}^{2}$. Let $\Gamma \subset \mathbb{P}^{2}$ be the image of $C$ under the $g_{n}^{2}$. The two pencils are cut on $\Gamma$ by the lines through two points $P, Q \in \mathbb{P}^{2} \backslash \Gamma$. Each of them corresponds to a line in the dual plane $\mathbb{P}^{2 \vee}$, that cuts the dual curve $\Gamma^{\vee}$ in a divisor whose pullback on $C$ is the corresponding jacobian divisor: then they are linearly equivalent.

(ii) By choosing a base-point-free $g_{n}^{1}$ in $|D|$ and a base-point-free $g_{m}^{1}$ in $|E|$ we can use them to define a birational morphism $C \longrightarrow \mathbb{P}^{1} \times \mathbb{P}^{1}$. By embedding $\mathbb{P}^{1} \times \mathbb{P}^{1}$ as a quadric $Q \subset \mathbb{P}^{3}$ and then projecting from a general $O \in Q$ we can map $C$ birationally to a plane curve $\Gamma$ of degree $n+m$ with two ordinary multiple points $A, B$, of multiplicity $n$ and $m$ respectively. The pencil of lines through $A$ (respectively $B$ ) cuts on $\Gamma$ the $g_{m}^{1}$ (respectively the $\left.g_{n}^{1}\right)$. Consider the dual curve $\Gamma^{\vee} \subset \mathbb{P}^{2 \vee}$. Then the lines of $\mathbb{P}^{2 \vee}$ cut on $\Gamma^{\vee}$ divisors of $\left|J_{D+E}\right|$. Consider in particular the line $r$ corresponding to the pencil through $A$. It cuts the divisor $J_{E}+2 D^{\prime}$, where $D^{\prime}$ is the divisor corresponding on $C$ to the branches of $\Gamma$ at $A$, because $r$ must be tangent to $\Gamma^{\vee}$ along $D^{\prime}$; by construction $D^{\prime} \in|D|$. Similarly the line $s$ of $\mathbb{P}^{2 \vee}$ corresponding to the pencil of lines through $B$ cuts the divisor $J_{D}+2 E^{\prime}$, where $E^{\prime} \in|E|$ is the divisor corresponding on $C$ to the branches of $\Gamma$ at $B$. Therefore $J_{E}+2 D^{\prime}, J_{D}+2 E^{\prime} \in\left|J_{D+E}\right|$ and this concludes the proof.

From the proposition it follows that the jacobian divisors of the bpf pencils contained in $|D|$ belong to the same complete linear series $\left|J_{D}\right|$ which is called the jacobian series of $|D|$. Moreover part (ii) of the proposition implies that for every bpf linear series $|D|$ the linear equivalence class $\left|J_{D}-2 D\right|$ is independent of $|D|$. It is called the canonical series and denoted by $|K|$. One defines the genus $g=g(C)$ by the identity

$$
\operatorname{deg}(K)=2 g-2
$$

It is not obvious that $g$ is an integer, i.e. that $J_{D}$ has even degree: this will be shown in a moment. Of course this definition agrees with the topological one, thanks to Hurwitz formula, but the approach taken here is purely algebraic. 
In modern terms, if $\varphi_{V}: C \longrightarrow \mathbb{P}^{1}$ is the morphism defined by $|V|$, the ramification divisor is the degeneracy divisor of the induced homomorphism $T_{C} \longrightarrow \varphi_{V}^{*} T_{\mathbb{P}^{1}}$, and therefore it has degree

$$
\nu=\operatorname{deg}\left(\varphi_{V}^{*} T_{\mathbb{P}^{1}}\right)-\operatorname{deg}\left(T_{C}\right)=2 n+2(g-1)
$$

and this of course agrees with (1).

The next step is to take as a birational model of $C$ a plane curve $\Gamma$ of degree $n$ with $\delta$ nodes and no other singularities and to compute the genus using the $g_{n}^{1}$ defined by the pencil of lines through a general point $O \in \mathbb{P}^{2}$. Now the degree $\nu$ of the branch divisor equals the degree of the dual curve, and this is computed by the first Plucker formula which gives:

$$
\nu=n(n-1)-2 \delta
$$

and this is an even number. Therefore we obtain:

$$
g=\left(\begin{array}{c}
n-1 \\
2
\end{array}\right)-\delta
$$

This is the so-called Clebsch formula. The jacobian divisor of the $g_{n}^{1}$ defined by the pencil of lines through a general point $O \in \mathbb{P}^{2}$ is cut on $\Gamma$ by the polar curve $\Gamma_{O}$ of $\Gamma$ with respect to $O$. More precisely, $\Gamma_{O}$ passes through the nodes with multiplicity one, and cuts elsewhere on $\Gamma$ the jacobian divisor. $\Gamma_{O}$ is a particular adjoint curve (of degree $n-1$ ). Therefore the adjoints of degree $n-3$ cut on $\Gamma$ canonical divisors. It follows that

$$
\operatorname{dim}(|K|+1) \geq\left(\begin{array}{c}
n-1 \\
2
\end{array}\right)-\delta=g
$$

i.e. $|K|$ is a $g_{2 g-2}^{g-1+\alpha}$, with $\alpha \geq 0$. We will prove that $\alpha=0$ (Corollary 4.3).

If the irreducible plane curve $\Gamma$ of degree $n$ has ordinary multiple points of multiplicities $m_{1}, \ldots, m_{\delta}$ respectively, then an argument similar to the case of nodes involving the polar curves gives the following generalization of the Clebsch formula:

$$
g=\left(\begin{array}{c}
n-1 \\
2
\end{array}\right)-\sum_{1}^{\delta}\left(\begin{array}{c}
m_{i} \\
2
\end{array}\right)
$$

We can now prove the following:

Theorem 2.2 (Riemann-Roch, weak form). If $|D|$ is a complete $g_{n}^{r}$ then $r \geq n-g$.

Proof. Take a plane model $\Gamma \subset \mathbb{P}^{2}$ of $C$ having degree $m$ and $\delta$ nodes and no other singularities. The adjoint curves of degree $d \geq m$ cut on $C$ a linear series $g_{m_{d}}^{r_{d}}$ with $m_{d}=m d-2 \delta$ and

$$
r_{d} \geq\left(\begin{array}{c}
d+2 \\
2
\end{array}\right)-\delta-\left(\begin{array}{c}
d-m \\
2
\end{array}\right)-1=m_{d}-g
$$

The given $g_{n}^{r}$ can be obtained as the series cut on $C$ by the adjoints of a sufficiently high degree $d$ passing through a suitable fixed divisor $F$ of 
degree $k$. But then $n=m_{d}-k$ and $r \geq r_{d}-k$. Comparing with the previous inequality we get $r \geq n-g$.

\section{An application of a formula of Schubert's}

Consider the symmetric product $C_{n}$, namely the quotient of the cartesian product $C^{n}$ of $C$ by itself $n$ times modulo the action of the symmetric group $\sigma_{n}$ permuting the factors. It is well known and elementary that $C_{n}$ is a nonsingular $n$-dimensional projective variety that can be set-theoretically identified with the set of all effective divisors of degree $n$ on $C$. Let

$$
\mathcal{D}_{n}=\{(D, x): x \in \operatorname{Supp}(D)\} \subset C_{n} \times C
$$

Then $\mathcal{D}_{n}$ is a divisor such that

$$
\mathcal{D}_{n \mid\{D\} \times C}=D
$$

for every $D \in C_{n}$. We will call $\mathcal{D}_{n}$ the universal (or tautological) divisor on $C_{n} \times C$. Let

$$
C_{n} \stackrel{q_{n}}{\leftarrow} C_{n} \times C \stackrel{p}{\longrightarrow} C
$$

be the projections. Given an invertible sheaf $A$ on $C$ such that $\operatorname{deg}(A) \geq n$, the $n$-th secant bundle of $A$ (on $C_{n}$ ) is $E_{A}=q_{n *}\left(p^{*} A_{\mid \mathcal{D}_{n}}\right)$; it is a vector bundle of rank $n$ on $C_{n}$. Given $U \subset H^{0}(A)$ of $\operatorname{dim}(U)=\ell+1 \geq 2$, we have a natural evaluation map:

$$
e_{U, n}: U \otimes \mathcal{O}_{C_{n}} \longrightarrow E_{A}
$$

If $0 \leq s \leq n$ we let $V_{n}^{s}(U) \subset C_{n}$ denote the subscheme defined by the condition $\operatorname{rank}\left(e_{U, n}\right) \leq s$. It is supported on the divisors $D \in C_{n}$ which impose $\leq s$ conditions to $|U|$. By general facts about determinantal varieties we know that every component of $V_{n}^{s}(U)$ has codimension $\leq(\ell+1-s)(n-s)$. In some cases equality holds.

Proposition 3.1. (i) Assume that $U \subset H^{0}(A)$ generates $A$, i.e. that $|U|$ is a base-point-free $g_{\operatorname{deg}(A)}^{\ell}$. Then $V_{\ell+1}^{\ell}(U) \subset C_{\ell+1}$ is a divisor.

(ii) Assume that $\operatorname{dim}(U)=2$ and $U$ generates $A$, i.e. that $|U|$ is a basepoint-free pencil. Then $V_{n}^{1}(U)$ is a curve for every $1 \leq n \leq \operatorname{deg}(A)$.

Proof. Left to the reader.

The following is the key enumerative result we will use:

Theorem 3.2 (Schubert [5]). Suppose that $V \subset H^{0}(L)$ defines a $g_{n}^{r}, r \geq 1$, and $W \subset H^{0}(M)$ defines a $g_{m}^{1}$ on $C$, with $m \geq r+1$. Let $g$ be the genus of $C$. Then the number of effective $D \in C_{r+1}$ that are simultaneously contained in a divisor of the $g_{n}^{r}$ and in a divisor of the $g_{m}^{1}$ is:

$$
Z_{r, n ; m}=\left(\begin{array}{c}
m-1 \\
r
\end{array}\right)(n-r)-\left(\begin{array}{c}
m-2 \\
r-1
\end{array}\right) g
$$


Here each $D$ counted by the formula must be taken with an appropriate multiplicity, which is one when $D$ is reduced and contained in unique reduced divisors of $|V|$ and of $|W|$. In modern terms $Z_{r, n ; m}$ is the intersection multiplicity of $V_{r+1}^{1}(W)$ with $V_{r+1}^{r}(V)$ in $C_{r+1}$. The proof of this theorem will be given in $\$ 5$. The following observation is the key step for the proof of the Riemann-Roch Theorem.

Proposition 3.3 (Castelnuovo [3]). Suppose that $V \subset H^{0}(L)$ defines a $g_{n}^{r}$, $r \geq 1$, and $W \subset H^{0}(M)$ defines $a g_{m}^{1}$ on $C$. If $n-r<g$ and $m \leq r+1$ then every divisor of $|W|$ is contained in a divisor of $|V|$ (shortly $|W|$ is contained in $|V|$ ). Moreover the divisors of $|W|$ impose $\leq m-1$ conditions to $|V|$.

Proof. Assume first that $m=r+1$. The assumptions imply that $Z_{r, n ; r+1}<$ 0 . This can only happen if $V_{r+1}^{1}(W) \subset V_{r+1}^{r}(V)$ because $V_{r+1}^{1}(W)=|W| \cong$ $\mathbb{P}^{1}$ is irreducible. Since $V_{r+1}^{1}(W)$ consists of all divisors in $|W|$, the first part of the proposition is true. Moreover it is clear that all divisors of $|W|$ impose at most $r=m-1$ conditions to $|V|$.

Assume now that $m<r+1$ and fix an arbitrary $F \in C_{r+1-m}$. Then, the linear series $F+|W|$ is a $g_{r+1}^{1}$, and $F+|W|=V_{r+1}^{1}(F+|W|) \subset C_{r+1}$. Since we again have $Z_{r, n ; r+1}<0$, we conclude as before that $F+|W| \subset V_{r+1}^{r}(V)$ because it is isomorphic to $\mathbb{P}^{1}$, hence it is irreducible. Moreover, since $F+|W|$ imposes at most $r$ consitions to $|V|$ and $F$ is arbitrary, it follows that $|W|$ imposes $\leq m-1$ conditions to $|V|$.

Remark 3.4. The proof can be easily modified to show that the same conclusion holds if $W \subset H^{0}(M)$ is a $g_{m}^{s}$, with $s \geq 2$.

Corollary 3.5. Suppose $|V|$ is a $g_{n}^{r}$ and $|W|$ a $g_{m}^{s}$ on $C$. Assume that $n-r<g$ and $m-s+1 \leq r+1$. Then $|W|$ is contained in $|V|$. Moreover $|W|$ imposes at most $m-s$ conditions to $|V|$.

Proof. We may assume that $s \geq 2$. Let $H$ be a general divisor of $|W|$. We have to show that it is contained in a divisor of $|V|$. For every choice of an effective divisor $G<H$ of degree $m-s+1$, the series $|G|$ is a $g_{m-s+1}^{1+\epsilon}, \epsilon \geq 0$, and by Proposition 3.3 and Remark 3.4 it imposes $m-s-\delta$ conditions to $|V|$, for some $\delta \geq 0$. Choose $G$ so that $\delta$ is minimum and let $x \in \operatorname{Supp}(G)$ be such that $G-x$ imposes the same number $m-s-\delta$ of conditions to $|V|$ : such an $x$ exists because $G$ does not impose independent conditions to $|V|$. By the choices made, for every $y \in \operatorname{Supp}(H-G)$, the divisor $G-x+y$ imposes at most $m-s-\delta$ conditions to $|V|$. This implies that every $D \in|V|$ containing $G$ also contains $y$, and therefore there is a $D \in|V|$ containing $G$ and all $y \in \operatorname{Supp}(H-G)$. Thus $H$ is contained in $D$. The last assertion is clear.

Remark 3.6. This proof is essentially the same given in [6], n. 71, in [3] and in [7], p. 293-294. 
Observe that using the enumerative formula (4) we have deduced an inclusion of linear series, i.e. a functional relation, from a numerical condition. Now we can easily proceed to the proof of the Riemann-Roch Theorem.

\section{The Theorem of Riemann-Roch}

Definition 4.1. A divisor $D$ (or a complete linear series $|D|$ ) is called special If $|D|$ is contained in the canonical series $|K|$.

We have the following

Corollary 4.2. Assume $g \geq 2$. If $D$ is an effective divisor such that $\operatorname{dim}(|D|)>\operatorname{deg}(D)-g$ then $|D|$ is special and imposes at most $\operatorname{deg}(D)-$ $\operatorname{dim}(|D|)$ conditions to $|K|$.

Proof. Suppose that $|D|$ is a $g_{n}^{r}$. We may assume $r \geq 1$ because otherwise $n<g$ and the conclusion is obvious. Applying Corollary 3.5 with the $g_{n}^{r}$ and the $g_{m}^{s}$ replaced by $|K|$ and $|D|$ respectively the conclusion follows immediately.

Corollary 4.3. $|K|$ is a $g_{2 g-2}^{g-1}$ and it is the only $g_{2 g-2}^{g-1}$ on $C$.

Proof. The first assertion is clearly true if $g=0,1$. Assume $g \geq 2$ and assume by contradiction that $\operatorname{dim}(|K|) \geq g$. Then the same proof of Corollary 4.2 applies to the linear series $|D|$ of dimension $\operatorname{deg}(D)-g$. This would imply the absurd fact that all linear series are special because, by Theorem 2.2 , every $D$ satisfies $\operatorname{dim}(|D|) \geq \operatorname{deg}(D)-g$. The uniqueness follows from Corollary 4.2 applied to any other $g_{2 g-2}^{g-1}$.

Theorem 4.4 (Riemann-Roch). If $|D|$ is a complete $g_{n}^{r}$ on $C$ then

$$
r=n-g+i
$$

where $i=H^{0}(K-D)=\operatorname{dim}(|K-D|)+1$.

Proof. By Corollary 4.2

$$
i-1=\operatorname{dim}(|K-D|) \geq g-1-(n-r)
$$

i.e. $i=g-n+r+\epsilon$, for some $\epsilon \geq 0$. By applying the same argument to $|K-D|$ we obtain:

$$
r=\operatorname{dim}(|D|)=g-1-(2 g-2-n-i+1)+\epsilon^{\prime}
$$

for some $\epsilon^{\prime} \geq 0$. By adding the two estimates we obtain:

$$
i+r=(g-n+r+\epsilon)+\left(-g+n+i+\epsilon^{\prime}\right)=r+i+\epsilon+\epsilon^{\prime}
$$

which gives $\epsilon+\epsilon^{\prime}=0$. But then $\epsilon=\epsilon^{\prime}=0$. 


\section{SCHUBERT'S FORMULA}

In this section we will prove Theorem 3.2. The formula (4) has been proved again by Castelnuovo in [3], n. 8. A modern version of it can be found in [1, p. 345. The proof given in [1] uses modern intersection theory, including Porteous formula and intersection theory on the symmetric product. Here we will reproduce the elegant simple proof given by Enriques in [4.

If $r=1$ we want to count the number $Z_{1, n ; m}$ of pairs of points that are simultaneously contained in a divisor of a given $g_{n}^{1}$ and a given $g_{m}^{1}$. Let us assume first that both pencils are bpf and let us make the simplifying assumption that their common pairs consist of distinct points and have disjoint supports. Then they define a morphism $\varphi: C \longrightarrow \mathbb{P}^{1} \times \mathbb{P}^{1}$ whose image is a curve of bidegree $(n, m)$ having $Z_{1, n ; m}$ nodes. Identifying $\mathbb{P}^{1} \times \mathbb{P}^{1}$ with a nonsingular quadric $Q \subset \mathbb{P}^{3}$ and projecting from a general point of $Q$ the curve $C$ is mapped birationally to a plane irreducible curve of degree $n+m$ having an ordinary $m$-fold point, an ordinary $n$-fold point and $Z_{1, n ; m}$ further nodes. By applying (3) we therefore have:

$$
g=\left(\begin{array}{c}
n+m \\
2
\end{array}\right)-\left(\begin{array}{l}
n \\
2
\end{array}\right)-\left(\begin{array}{c}
m \\
2
\end{array}\right)-Z_{1, n ; m}
$$

which gives

$$
Z_{1, n ; m}=(n-1)(m-1)-g
$$

and this is precisely (4) for $r=1$. Now assume that $|V|$ is a $g_{m}^{1}$ with a fixed point $P$. Then it has in common with the $g_{n}^{1}$ the $Z_{1, n ; m-1}$ pairs common to the $g_{n}^{1}$ and $|V(-P)|$, plus the pairs consisting of $P$ and any point different from $P$ of the divisor of the $g_{n}^{1}$ that contains $P$. Therefore we have again

$$
Z_{1, n ; m-1}+n-1=Z_{1, n ; m}
$$

common pairs. By iterating this argument one takes care of pairs of pencils with any number of base points.

Let us now consider a $g_{n}^{r}$ and a $g_{m}^{1}$, both bpf. Let us add a point $P$ to the $g_{n}^{r}$ as a fixed point and denote by $g_{n}^{r}+P$ the $g_{n+1}^{r}$ thereby obtained. Let us assume that $P$ has been chosen not in any of the $(r+1)$-tuples common to the $g_{n}^{r}$ and the $g_{m}^{1}$. Then the number of $(r+1)$-tuples common to $g_{n}^{r}+P$ and to the $g_{m}^{1}$ are counted as follows. There are $Z_{r, n ; m}$ of them that are those common to the $g_{n}^{r}$ and the $g_{m}^{1}$. Then there are $\left(\begin{array}{c}m-1 \\ r\end{array}\right)$ that consist of $P$ plus any $r$ points of the unique divisor of the $g_{m}^{1}$ containing $P$ (and are therefore contained in a unique divisor of the $g_{n}^{r}$ ). Hence $g_{n}^{r}+P$ and the $g_{m}^{1}$ have

$$
Z_{r, n+1 ; m}=Z_{r, n ; m}+\left(\begin{array}{c}
m-1 \\
r
\end{array}\right)
$$


$(r+1)$-tuples in common. By iterating we obtain:

$$
Z_{r, n+h ; m}=Z_{r, n ; m}+h\left(\begin{array}{c}
m-1 \\
r
\end{array}\right)
$$

as the number of $(r+1)$-tuples common to $g_{n}^{r}+P_{1}+\cdots+P_{h}$ and the $g_{m}^{1}$, where $P_{1}, \ldots, P_{h} \in C$ are subject to the same condition as $P$ was.

Now we compute the number of $(r+1)$-tuples that are simultaneously contained in a divisor of the $g_{n}^{r}$ and in a divisor of the $g_{m}^{1}$ as follows. Denote by $|W|$ the $g_{n}^{r}$ and consider a bpf pencil $|V|$ contained in it. Let $r|V|$ be the $g_{r n}^{r}$ which is the minimal sum of $|V|$ with itself $r$ times; equivalently $r|V|$ is the pullback of the composition of $\varphi_{V}: C \longrightarrow \mathbb{P}^{1}$ with the $r$-th Veronese embedding of $\mathbb{P}^{1}$ in $\mathbb{P}^{r}$. Also consider the series $|W|+D_{1}+\cdots+D_{r-1}$, where $D_{1}, \ldots, D_{r-1} \in|W|$ are general divisors. Then $|W|+D_{1}+\cdots+D_{r-1}$ is another $g_{r n}^{r}$. Since both $r|V|$ and $|W|+D_{1}+\cdots+D_{r-1}$ are contained in the same complete series $|(r-1) W|$ we may assume that they have the same number of $(r+1)$-tuples in common with the $\left.g_{m}^{1} \cdot 1\right]$

The number of $(r+1)$-tuples common to $|W|+D_{1}+\cdots+D_{r-1}$ and the $g_{m}^{1}$ is given by the formula (6) with $h=(r-1) n$. On the other hand the $(r+1)$-tuples common to $r|V|$ and to the $g_{m}^{1}$ are all obtained by choosing a pair $Q_{1}+Q_{2}$ common to $|V|$ and to the $g_{m}^{1}$ plus any $r-1$ of the remaining points of the unique divisor of the $g_{m}^{1}$ that contains $Q_{1}+Q_{2}$. Therefore they are in number of

$$
Z_{1, n ; m} \cdot\left(\begin{array}{c}
m-2 \\
r-1
\end{array}\right)
$$

After recalling (5) and comparing with (6) with $h=(r-1) n$ we obtain:

$$
Z_{r, n ; m}+(r-1) n\left(\begin{array}{c}
m-1 \\
r
\end{array}\right)=[(n-1)(m-1)-g]\left(\begin{array}{c}
m-2 \\
r-1
\end{array}\right)
$$

and this is clearly equivalent to (4).

Acnowledgements. Research partially supported by the project MIURPRIN Geometria delle varietà algebriche. The author is member of GNSAGAINDAM.

\section{REFERENCES}

[1] Arbarello E., Cornalba M., Griffiths P., Harris J.: Geometry of Algebraic Curves I, Springer Grundlehren b. 267 (1984). 5

[2] Brill A., Noether M.: Ueber die algebraischen Functionen und ihre Anwendung in der Geometrie. Math. Ann. 7 (1873), 269-310. 1

[3] Castelnuovo G.: Ricerche di geometria sulle curve algebriche, Atti R. Accad. Sci. Torino 24 (1889), 196-223. 1, 3.3, 3.6, 5

[4] Enriques F.: Questioni numerative e loro significato nella geometria sopra le curve algebriche. Rend. Acc. Naz. Lincei (5) 28 (1919), 370-374. 1, 5,1

[5] Schubert H.: Kalkul der Abzahlenden Geometrie, Teubner (Leipzig), 1874. 3.2

\footnotetext{
${ }^{1}$ This is the key enumerative assumption made by Enriques in [4, which can be fully justified in terms of intersection theory on the symmetric product $C_{r+1}$.
} 
[6] Segre C.: Introduzione alla geometria sopra un ente algebrico semplicemente infinito. Annali di Matematica Pura e Applicata (2) 23 (1894), 41-142. 1.3 .6

[7] Severi F.: Trattato di Geometria Algebrica - Volume I Parte I: Geometria delle serie lineari. Zanichelli (Bologna), 1926. 1, 3.6

[8] Severi F.: Prefazione. In Corrado Segre: Opere, vol. I. Cremonese (Roma), 1957. 1

E-mail address: sernesi@mat.uniroma3.it

Dipartimento di Matematica e Fisica, Università Roma Tre, Roma. 